en effet obtenues, dans ces expériences, au moyen d'un robinet dont l'action était comparable à celui d'une vanne d'étranglement.

Ce qui précède fait aussi voir les précautions qu'il est indispensable de prondre lorsque l'on emploie les vannes d'étranglement pour les hautes chutes.

La valeur (3I) de $\lambda$ 'montre en effet que l'action de ces vannes ne devient scnsible que lorsque la section $\lambda s$, réduite par la vanne, devient comparabie à la section $\sigma$ du distributeur, mais, à partir de cet instant, c'est dans un temps excessivement court que la fermeture totale se produira et, par suite, ce temps étant très généralement inférieur à celui du coup de bélier direct, ce coup de bélier se produira avec l'intensité correspondant aux fermetures brusques, bien que la vanne ait pu être fermée assez lentement. La seule différence qu'il pourra y avoir, c'est que le coup de bélier au lieu de devenir brusquement égal à $\frac{a r_{0}}{g}$ croîtra très rapidement de o à cette valeur; on se retrouvera dans le cas d'une fermeture dont la durée est plus faible que celle du coup de bélier direct sans être absolument brusque.

Ce sont bien les résultats qu'ont donné les expériences de St-Henri. Com'e De Sparre.

\section{Les Voies françaises d'accès au Simplon et le Percement du Mont Blanc}

Maintenant que le percement du Simplon est un fait accompli, on commence à s'inquiéter sérieusement, un peu tard il est vrai, des conséquences que l'exploitation de la nouvelle ligne internationale amènera dans le trafic de nos chemins de fer. Diverses solutious ont été proposées. Il n'appartient pas à la Houille Blanche de prendre fait et cause pour l'une ou pour l'autre, mais il lui a ppartient d'ouvrir ses colonnes à la plus large discussion... d'où jaillit la lumière. Nous avons publié, en juin rgo4, un extrait d'une étude des diverses voies de commu. nications à étahlir entre la France, la Suisse et l'Italie due à M. lingénieur Ph. Stéphant. Cet exırait portait sur la ligne du Mont Blanc. M. Stéphani explique aujourd'hui les raisons qui, selon lui, mili ent plus particulièrement en faveur de cette nouvelle voie d'accès à travers les Alpes.

N. D. L. R.

La mise en exploitation prochaine de la nouvelle ligne du Simplon affecte au plus haut point les intérêts de Ja France et du commerce français; elle sera même de nature à les léser gravement lorsque la percée du Simplon sera directement mise en communicalion, à lravers les Alpes Bernoises, avec le centre et le nord de la Suisse, l'Alsace-Lorraine et l'Allemagne du sud.

La Jigne du Sarrit-Gothard a enlevé aux lignes françaises le trafic de la vallée de la Moselle el de la mellleure partıe de la kelgique, en portant alnsi à notre commerce un préjudice fort appréciable.

On a dit que le percement du Simplon étalt la grande revanche française du Gothard. Nous ne voyons pas trop sur quels raisonnements est basée cette fausse légende. La vérılé, c’est que le Simplon rendra plus actives les relations économiques entre l'Ilalie du nord, la Suisse occidentale et l'Allemagne du sud.

La France ne pourra tırer une utilıté appréciable du Simplon qu'en créant à grands frais, à travers le Jura, de nouvelles voies d'accès aux lignes suisses se dirigeant sur le Simplon, ou en amélinrant tout au moins celles existantes (projet Saint-AmourBellegarde et Lons-le-Saunier, La Faucille, Genève, Annemasse; rectificalion Frasne-Vallorbes, Bellegarde, saint. Gingolph, elc.).

Depuis longtemps on envisage avec anxiété le tort considérable que va nous causer la concurrence de ce nouveau Gothard. D'aucuns s'en s'ont occupés, de nombreuses commissions ont été nommées, maints rapports ont été déposés, bien des propositions ont élé proposées pour venir contrebalancer tse préjudıce prochain.

L'opinion publique française se préoccupe vivement, et à bon droil, de la question des voies d'accès au Simplon, mais bien a tort le débal a élé presque exclusivement cantonné, jusqu'à ce jour, sur le point de savuir quel estle mellenr chnix a faire entre la Faucille ou la Frasne-Vallorbes, alors quill serait, a nolre point de vue, beaucoup plus important de savoir si une nouvelle percée des Alpes entre la France et l'Italie ne répondrat pas davantage aux ınlérêts françaıs, et s'il ne conviendrast pas mirux à la France dappliquer à cetle œuvre exclu-ivement francoitalienne, l'effort financier, peut-être plus considérable, qu'elle seraıt oblıgée de faıre pour tırer viaıment utılıté du Sımplon, el en procurer une bıen plus grande el surlout plus certaıne à la Sulsse et aux chemins de fer sunsses.

A celégard, une comparaıson sérıeuse s'impose entre le Sımplon en gúnérál et ses roles d'accès en particulier d'une parl, et la question quı a déjà donné lıeu, depuis longlemps. à de la borleuses études, d'une hgne internationale franco-italıenne au moyen de la percée du Mont Blanc d'autre part.

On nous répondra que le percement du Monl Blanc est une entreprise colossale qui demandera beaucoup de temps avant qu'on pursse la meltre a exéculion, si toutefois ce projel était pris en considéralion, et que pendant ce temps le Simplon détournera de nos ligues françalses, en faveur des lignes sursies el allemandes, la meilleure part du Irafic de Mlalie el de l'Orient, et que par conséquent, pour parer à cet inconvement, l'ıntérêt de lá France est de chercher une solution rapide, en créant une vole d'accès au Simplon, afin de conserver sur nos chemins de fer une part de cel important trafic.

Il s'agit de savoir si l'exéculion des solutions proposées exige moins de temps que la percée du Mont Blanc, et surtoul de savorr sı elles présentent les mêmes avantages que ce deruier.

La ligne du Simplon sera ouverte incessammenl à l'exploitation. et du rôlé français aucun décisıon n'a enıure été prise pour relier nos chemıns de fer à celle nouvelle ligne internationale.

Du côlé suisse et italien les vores d'accès ont été construites el quelques-unes d'elles (la ligne d'Arona-Dumodossola el le tronçon Santhià-Borgomanero destıné à relıer Turın au Simplon) sont déja ouvertes a l'explontation.

L'ouverlure à l'exploitation du Simplon est un événement d'une importance économique considérable et dont ll est difficile d'évaluer toutes les conséquences.

Non seulement la nouvelle ligne, beaucoup plus avantageuse sous tous les rapports que celles du Mont Cenis et du Golbard, allirera à elle une partıe de leur trafic, mais encore gràce à des tarifs plus avantageux et à des communicalions plus rapides, elle créera des courants nouveaux dont nos concurrents seroutl les premiers à en profiler.

Les Belges et les Allemands verront, grâce à cette nouvelle voie, augmenter dans de fortes proportions le chiffre de leurs échanges commerciaux.

Qu'avons-nous falt en France pour parer à cette redoutable concurrence? Rien jusqu'a présent.

Divers projets ont été mis en avant. Actuellement, ainsi que nous l'avons dit plus haul. la question est cantonnée sur deux projets concurrenls : la Frasne-Vallorbes et la ligne Pontarlier-Genève par la percée du Jura.

La Frasne-Vallorbes améliore les conditions actuelles d'exploiLation de la ligne par Pontarlier, au point de vue climatérique, en abaissant à 894 mètres l'altıtude maxima de la traversée ru dernier chaînon đu Jura, qui est aujoura'hui de 1.012 mètres, près de Jougue, et au poini de vue du profil, en substıtuant des déelivilés de $15 \mathrm{mms}$ à celles de $25 \mathrm{mms}$ existánt entre Pontarlier el Vallorbes, mais il laisse subsister les déclivités de $20 \mathrm{mms}$ entre Mouchard et Vallorbes et entre Vallorbes et Dallens.

Mais si l'on attend que les Bernors aient percé les Alpes Bernoises soil au Locthsberg ou ai Wilsdrubel, tout l'intérèt de lit Frasne-Vallorbes disparait.

La distance entre Paris et Milan par Dijon-Pontarlier-les.Verrières-Neuchatel-Berne (Locthsberg-Sımplon) sera de $836 \mathrm{kms}$.

Celle entre Paris el Milan par Dijon-Pontarlier-Frasne-VallorbesSimplon sera de 830 kilomètres.

La Frasne-Vallorbes ne raccourcira donc la distance ParisMilan que de 6 kllomèlres seulement. D'un côté comme de l'autre il faut suivre un territoire étranger et il est tout à fait indifférent pour la France que le trafic se fasse par Neuchatel, Berne, Brigue ou par Lausanne, Bouveret, Brigue. 
Dans tous les cas, la construction de la ligne Frasne-Vallorbes ne peut avoir qu'un intérêt partiel pour le commerce lranẹis. Fle l"intéresse directement que le Nord et l'Est de la France.

or, quand on parle d'ulilité nationale, 1 faut associer le pays, al non pas seulement une zone, dans toute la mesure où sa conficuralıon géographıque le permet, aux résultats d'une entreprise ilont il est appelé a se répartir les charges.

Le trafic francals, avec sa puissance d'expansion et d'absorption, niest pas lucalisé dans un triangle imaginatre qui aurast une ligne allant rle Dieppe a Dunkerque comme hase et dont Je sommet serait à Delle. Ce triangle ne représente en surface que la cinquième partıe à peine du terriloıre.

Il laul coustaler en outre que la rég̣ion de Dieppe à Dunkerque et les ports du Norl, ainsi que nolre frontière del'Est, trouveront satisfaclion dans le Loclhsberg ou le Wildstrubel pour profiter du Simplon vers l'dlalie et ses au delà, sans quil soit nécessaıre de dépenser 27 millions pour la Frasne-Vallorbes.
Cette nouvelle lıgne de Lons-le-Saulnier à Genève consiste en un tracé d'une longueur à construire entre les stations de Lons-leSaulnier et de Meyrın de 73 kilomètres, non compris les parties communes empruntées dans les gares exislantes de Lons-leSaulmer, Saml-Claude el Meyrm.

On traverserait presque en plan la chaine principale du Jura, non plus au Col de la Faucille mais entre le Colombier de Gex et le Reculet. Ce projet comprend trois lumnels ifur mesurent respeclivement 6400 mètres, 11400 mètres et 15200 mètres.

La dénense serait. d'après les études accomplies par la Compagnie P.-L -M., de 120 à 130 million;.

Il est vial que Genève y contribuerait pnur sat part. Seulement les 20 millıons que le canton de Genève a oflert pour la constructıon de celte ligne sont tout à fait insulfisanls, sı l'on considère surtout les avaulages énormes qu'll est appelé à en retırer. Io projet de la Fancille, pr.sposé et délendu par le comilé Genevors, présente un intérêt tout partıculier puur la vılle de Genève, quı y trouveralt le moyen de réalıser enfin son ambition séculaire d'étape naturelle et dès lors presque obligée, pour les voyageurs se rendant de Dijon, Parıs et au delà en Itahe, ou viceversa. Il est par conséquent tout naturel que le concours financier de Genève pour la conslruction de la Faucille soil en proportion des avanlages qu'elle en relırera. A la vérıté celle lıgne a plus d'inlérêt pour la suisse et pour Genève que pour la France.

En oulre de la dépense très élevée que comporterait celte entrepirise, il faut, tenı' comple du lemps, très dıfficile a détermıner, que réclamera sa construclion. Sur le parcours de $75 \mathrm{kllo}$ mètres a construire entre Lons-le-Saulnier. plus le la moitıé, 39 kılomètres, dorvent être en tunnels.

Denx d'entre eux ont, l'un plus de 12 kilomitres, et l'autre plus de 15 kilometres. Le premier a la longueur du Mont Cenis et le second celle du Saınt-Gothard. En supposant que ces lumnels ne rencontrent pas des difficultés, ce seront des œuvres énos mes quı exigeronl plusıeuı s années.

Nous ne sommes certes pas opposé à la réalisation de ce projel que nous reconnaissons d'un intérêt capılal, non comme vole d'accès au Simplon mais comme voie daccès au Mont Blanc.

Mème si la dépense pour la France doit dépasser celle qu'exigerait, par exemple, la constructîn de la ligne Saint-dmour-Bellegarde ou la ligne Lons-le-Saulnier-Bellegarde, ne conviendrait-1l pas mieux de construire une de ces lignes qui ofrrirail au mons l'avantage d'un trajet effectué tout sur rals françars et nous eviterait ansi les ennuis d'une douane internationale en lemps de paix et le danger d'une neutrahlé peu rassurante en temps de guerre.

\section{Mrillan}

Si l'adoption du projet Frasne-Vallorbes offre quelque avanlage aux ports de la Manche et aux régions du Nord et de l'Est, 11 l'est d'aucune ulihté pour les ports de l'Atlantique et les régions de l'Ouest et du Centre.

Louverture du Golhard a eu une influence considérable pour la prospérité des ports belges et allemands.

ll est à crandre que le Simplon ne devienne une deuxième voie de pénétration en Italıe, à l'avanlage principal de l'Allemagne.

Celle considération dolt, a notre avis du moins, primer toutes les aulres. Une nouvelle ligne à travers les $\Lambda$ lpes, entre le Mont Cenis et le Simplon, mettant en communication directe le Centre de la France avec l'Italie, peut seule sauvegarder les intérêts de ces régions.

On a proposé comme remède à cet inconvénient la construction de la ligne Lons-le-Saulnier-Genève dite de la Faucille. Certes la ligne projetée de Lons-le-Saulnier-Genève est, au point de vue de la commodité, celle qui résourraut le plus favorablement la question d'accès au Simplon. En effet, celte ligne dont la cote n'excède pas 559 mètres et les rampes ne dépassent pas le 10 pour 1000 offrirait les meilleures condilions pour une ligne a granl lrafic entre Paris et Milan, mais comme la première elle nintéresse qu'une parlie du territuire : le Centre et l'Ouest.

D'autre part son exéculion présente de sérieuses difficultés.
Au lieu re perdre son temps à discuter pour savoir quelle sera la meılleure vole d'accès au sımplon, on ferait beaucoup mieux d'éludier le projet du Mont Blanc qui présente, sur la pluparl des divers projets de voies d'accès au simplon, un intérêt majeur évident, et qui, d'autre part, constilue pour la France et les chemins de fer français, l'unique moyen de réparer le préjudice certaın quı, à côlé de quelques avanlages chèrement achelés, leur. sera apporté dans l'avenir surtout par la percée du simplon.

Seule, la ligne du Mont Blanc permettrait aux compagnies de chemins de fer français de faire une concurrence efficace soit à la ligne du Gothard (Lratic anglo-italien et italo-belge), soit à la vole en perspective Ostende-Vienne-Salonique (tratic de l'Angleterre en Orient et pour le service de la malle des Indes).

Il ne faut pas perdre de vue que le Simplon, ainsi que ses voies d'accès, sur un long parcours, sont sur terre étrangère, alors que le Mont Blanc est sur la frontière franco-italienne el que ses voies d'accès du côté nord-ouest sont sur territoire français.

Tous les ingénieurs compélents qui ont étudié le Mont Blanc dans le but d'y installer une ligne ferrée internationale ont reconnu que la percée da géant des Alpes présente les plus graudes facililés d'exécution, el que la voie peut être installée dans d'excel. lentes conditions de rampes et de courbes.

Le prolongement de Chamonix à Aoste en traversant le Mont 
Blanc, offrirat la communicalion la plus courte el ja plus directe qui puisse être élablie de Londres et Parıs a Turın et Mılan, el cela par rails francass jusqu'a la trontère ilalienne, saul' l'enclave de 15 kilomètres sur le lerrilore de Genòve. Ce serult un raccourci considérable cur l'llinéralre vià Mont Cenis et vià Simplon, et la suppression du long parcours sur les ralls suisses.

Linle d'une vore ferrée à travers to Mont Blanc ne date pas d'aupourd'hu : elle remonte à 1860. Le Gouvernement fraçais avant, à celte éporque, chargé M. Gorlin de Léprnay, ingénieur en chet les Ponts et Chaussées, d'étudier les voles de communicaIIon enlre la France el le Pímont. On n'a pas cessé depuis de s'uccuper de celte que-tion, el lout récemment le Ministère des Travaux Publics françals ellvovalt dans la vallíe d'Aoste M. B. Jacquier, ıngéureur en chef des ponts el Chaussées, pour l'éludier à nouvenu.

L'avanlage du Mont Blanc exicte dans la hauleur de ses accòs et la co nmodule que présentenl ses valléss pour alleındre jusqu'a la hauteur le son pied. qui, pour des éparsenrs égalea, est mlérleure à celle de lous les autres passages des Alpes; le massif forme une muralle de 3500 mètres de hauleur sur 13 kilomètres d'éparsseur. qui sépare les vallées de l'arve el de la Dorre; ces ruvieres en descendent avec les pentes Irès crdinares.

La ligue pourrat parlir de Sainl Gervars, remonter jusqu'à Chamonix el sortir à Courmayrur. L'énergie cnnsidérable que pourrait produire la Dotre serviral fout dabord aux divers travaux de percement dı lunnel el ensulte à fournır l'énergie électrıque nécescarre à mouvorr les trans. Muis d'autres tracés sont possibles: celu de M. de Lépluay, par evemple, donne de lrès fábles déclivités; 11 sélève de la vorle daccès à la pente de 13,5 mms jusqu'à Courmayeur, d'où la galerı longue de $13 \mathrm{k}$ l. 500 seulement, pasue à la cote 1000 sous la Dent du Gíant, et débouche un peu en aval le chamouix.

En résumé, les éturles léfà lalles démontrent que la ligne GènesMont-Blanc-Geneve-Dijon est supérieure a tous les autres projels pour fachiter les relations entre la France et litalie, el surtout au point de vue du transil enl re l'angleterre, les Indes el l'orient. Celte lınne nintéresse pas seulement une ou plusieurs rérions, mais elle intéresse la France cntière. Volla son grand avantage.

On peut évaluer à 2700000 franes par kilomèlre le prix de revient rles souterrains dans le genre de ceux dı Mont Blanc et a 407000 rranes par kilomèlre pour les autres passages a ciel ouvert et les peliles galeries.

La répense lolale pour l'élabissement du tunnel serait donc de 120 millıons de rancs y compris les lıgnes d'accès à construire dans les vallées de Cluses en France el d'Aoste en Italie.

Cetle ligne devrat être élablie moitió sur lerrloire français et mollé sur territolre vlalien. L'Italie, qui a largement coutribué au nercement du Gothard et du simplon, ne refuserait certamement pas son concours au Mont. Blanc, d'aulant plus que celle voie est d'une importance capilale, nous pourrions dire même d'une nécessilé absolue pıur Turın, le Piémont el Gênes. D'autre part, Genève ne refuserait pas certainement son concours, car la conatruchion de celle ligne iéalıseratt mıeux que lout aulre son ambitıon de devenir centre de Irafic. (SI la Suisse refusail Je concours nécessatre, on n'aurall qu'à dólourner le tracé et faire sulvre à la vole ferrée une ligne interteure à la frontière francaise, ce qui lasserait l'Helvétie complètement en dehors d'un immense rayon d'aciıon.) De sorte qu'à la France n'incomberait qu'une partie seulement de la dépense de 1 ?0 mullions exigés par l'exéculion de la percée du Mont Blanc. Celte dévense pourrait done être répartie enlre la France, l'italie et la Sursse. Au besoin, l'Angleterre qui s'intéresse beaucoup à ce tracé, lequel donnerait la ligne la plus rapule que l'on puisse imagıner de Londres à Brindisi, pourrait bien y contribuer nour sa part.

Si l'on considère les avanlages qu'on relirera d'une ligne de ce genre, la somme exıgée pour sa construclion est bien modeste par rapport aux sommes dépensées, ou à dépenser, pour d'aulres travaux analogues qui ont coûté, ou qui coûteraient, bien plus chers et qui n'offrent certrinement pas les conditions avantageuses qu'offre le tunnel da Mont Blanc.

De l'avis de tous les hommes compétents qui ont étudié cette ligne le capital employé dans cette entreprise serait largement rémunéré.

Il serait par conséquent souhaitable que la question du Mont Blanc soil de nouveau mise à J'élude sans plus de re!ard. L'avenir de notre commerce d'expansion, et de nos relations internationales, dépend de l'exécution de ce projet.

Ph. Stephani, Ingénieur.
PosT-Scriptum. - Gel artıcle élail déjà composé lorsque nous avons appris que M. Gauthier, ministre des Travaux Publics, s'élant déclaré en laveur du percement de la Faucille el du Mant Blanc. Nous applaudsssons de bon cœur le projet de M. Gaulhier, qui fail le plus graud honneur à con esprit d'inilialive. Par la réalisalion du projel de $M$. Gauther, et par la construclion de la lione Con-Nice, dont le premier coup de pioche ne tarlera être domné, la France posséderat la plus belle choîne de communications que l on puisse rêver enl re l'anglelerre, Paris, Mulan, Brinusi el lorreml. Celle immense artère amoindrirant considi. rablemenl les deux arteres parallèles du Gothard et du Sumplon qui viennent d'ètre créées, el qui menacent de détourner, qus détournent déjà, au profil de la Sursse allemande é de l'alle. magne thénane, une grande partie da trafic international.

P. S.

\section{ÉTUDE SUR LES}

\section{BHRRAGES EN MAÇONNERIE} et Murs de Réservoirs

(suile)

Barrage du Gouffre d'Enfer. - Ce barrage lerme la vallée du Furens au défilé du Goulfre d'Enfer. On l'appelle encore barrage du Furens ou de Rochetaillée, du nom de la localilé où il se trouve. Son couronnement se trouved 52 mètres de hauteur au-dessus du rocher', du côlé amont, et à 56 mètres du côté aval; il a un développement de $100 \mathrm{~ms}$ et est disposé en plan suivant un arc de cercle de 252 ms do rayon.

Il a été construit dans un double but: $1^{\text {e }}$ Emmagasiner leau surabondante pendatit la saison pluvieuse pour la restituer ensuite pendant la saison sèche, afin daugmenter le débit d'étiage du Furens et dassurer, en toute saison, lalimentation en eau de la ville de Saint-litienne; $2^{\circ}$ atténuer l'effet dévastateur des crues.

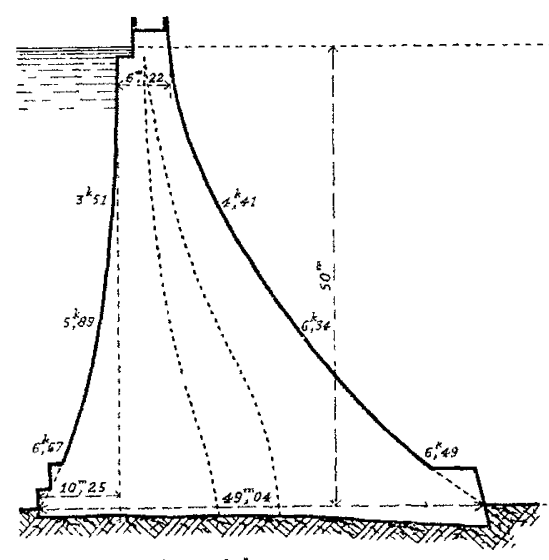

Fig. 14.

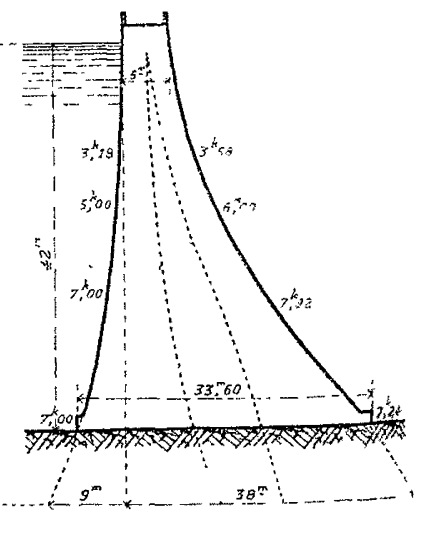

Fig. 15 .
La hauteur maxima de la retenue ne doit pas dépasserl 50 mètres au-dessus du fond amont. Primitivement, ainsi que nous l'avons déjả indiqué au commencement de celarticle, on avait préru qu'une tranche d'eau de $5 \mathrm{~m} 50$, correspon. dant à un volume de $400000 \mathrm{~m}^{3}$, devait toujours se trouver libre pour l'emmagasinement des crues, de sorte que le niveau maximum des eaux, en service normal, devait se trouver à $44 \mathrm{~m} 50$, créant ainsi une réserve de 1200000 mètres cubes Depuic, le niveau normal a été porté à $47 \mathrm{~ms}$ et, actuel. lement, il affleure la base du mur de garde qui surmontele corps du barrage proprement dit.

Ce mur de garde, qui est reporté du côté aval, a 5 métres de hauteur, $3^{\mathrm{m}} 75$ de largeur à la base et 3 mètres all sommet. 